\title{
Interactions between gastric microbiota and metabolites in
} gastric cancer

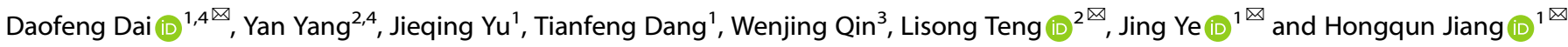

(c) The Author(s) 2021

The development and progression of gastric cancer (GC) is greatly influenced by gastric microbiota and their metabolites. Here, we characterized the gastric microbiome and metabolome profiles of $37 \mathrm{GC}$ tumor tissues and matched non-tumor tissues using 16s rRNA gene sequencing and ultrahigh performance liquid chromatography tandem mass spectrometry, respectively. Microbial diversity and richness were higher in GC tumor tissues than in non-tumor tissues. The abundance of Helicobacter was increased in non-tumor tissues, while the abundance of Lactobacillus, Streptococcus, Bacteroides, Prevotella, and 6 additional genera was increased in the tumor tissues. The untargeted metabolome analysis revealed 150 discriminative metabolites, among which the relative abundance of the amino acids, carbohydrates and carbohydrate conjugates, glycerophospholipids, and nucleosides was higher in tumor tissues compared to non-tumor tissues. The targeted metabolome analysis further demonstrated that the combination of 1-methylnicotinamide and $\mathrm{N}$-acetyl-D-glucosamine-6-phosphate could serve as a robust biomarker for distinction between GC tumors and non-tumor tissues. Correlation analysis revealed that Helicobacter and Lactobacillus were negatively and positively correlated with the majority of differential metabolites in the classes of amino acids, carbohydrates, nucleosides, nucleotides, and glycerophospholipids, respectively, suggesting that Helicobacter and Lactobacillus might play a role in degradation and synthesis of the majority of differential metabolites in these classes, respectively. Acinetobacter, Comamonas, Faecalibacterium, Sphingomonas, and Streptococcus were also significantly correlated with many differential amino acids, carbohydrates, nucleosides, nucleotides, and glycerophospholipids. In conclusion, the differences in metabolome profiles between GC tumor and matched nontumor tissues may be partly due to the collective activities of Helicobacter, Lactobacillus, and other bacteria, which eventually affects GC carcinogenesis and progression.

Cell Death and Disease (2021)12:1104; https://doi.org/10.1038/s41419-021-04396-y

\section{INTRODUCTION}

Gastric cancer (GC) is a prominent malignant tumor worldwide, particularly in Asia [1]. According to the latest cancer statistics in China, GC was responsible for over 679,100 new cases and 498,000 deaths in 2015, making it the second most frequently diagnosed cancer as well as the second leading cause of deaths related with cancer [2]. Infection with Helicobacter pylori is widely regarded as a high-risk factor for the development of GC as the majority of GC cases can be related to $H$. pylori [3]. Other risk factors include smoking, gender, and the consumption of smoked and high-salt foods [4].

Due to the acidic environment of the human stomach, it was previously believed that the stomach was not suitable for the growth of other microorganisms and was exclusively colonized by H. pylori. However, advances in sequencing technology have proven that the stomach is inhabited by a robust microbiota [5]. Previous studies have found that the microbial diversity in patients with intestinal metaplasia and GC were significantly decreased compared to in patients with superficial gastritis $[6,7]$. Moreover, other studies showed that GC was associated with increased microbial diversity and richness [8, 9]. A study using high-throughput sequencing techniques on a cohort of 276 Chinese patients with GC found that bacterial diversity and richness were lower in peritumoral and tumoral tissues than in non-tumor tissues, and that the composition of gastric microbiota was significantly altered in the different stomach microhabitats [10].

The development of tumors is affected not only by microbiota but also by their metabolites [11]. Kaji et al. used capillary electrophoresis time-of-flight mass spectrometry to quantify 93 metabolites in cancer and adjacent non-cancerous tissues from 140 patients with $G C$, revealing that $\beta$-alanine was both a significant predictor of peritoneal recurrence and a prognostic factor for GC [12]. Additionally, other studies have investigated the metabolite differences between non-tumor and cancerous tissues from patients with GC [13-15]. However, the number of metabolites quantified in these studies were very small, resulting in a lack of information on a large number of metabolites. Thus, further studies analyzing more metabolites are urgently needed.

\footnotetext{
'Jiangxi Otorhinolaryngology Head and Neck Surgery Institute, Department of Otorhinolaryngology Head and Neck Surgery, The First Affiliated Hospital of Nanchang University, Nanchang, Jiangxi, China. ${ }^{2}$ Department of Surgical Oncology, The First Affiliated Hospital, School of Medicine, Zhejiang University, Hangzhou, Zhejiang, China. ${ }^{3}$ Human Genetic Resources Center, The First Affiliated Hospital of Nanchang University, Nanchang, Jiangxi, China. ${ }^{4}$ These authors contributed equally: Daofeng Dai, Yan Yang.

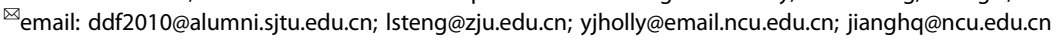
Edited by Dr Wei Jia
}

Received: 14 May 2021 Revised: 2 November 2021 Accepted: 10 November 2021

Published online: 24 November 2021 
Table 1. Clinicopathological characteristics of patients with GC in this study.

\begin{tabular}{|c|c|c|}
\hline Characteristics & Cohort 1 & Cohort 2 \\
\hline Total number & 37 & 20 \\
\hline \multicolumn{3}{|l|}{ Gender (no.) } \\
\hline Female & 11 & 6 \\
\hline Male & 26 & 14 \\
\hline Age (years, mean $\pm S D$ ) & $66.30 \pm 10.44$ & $62.20 \pm 13.74$ \\
\hline Weight $(\mathrm{kg}$, mean $\pm \mathrm{SD})$ & $60.72 \pm 11.82$ & $56.10 \pm 9.23$ \\
\hline Height $(\mathrm{cm}$, mean $\pm \mathrm{SD})$ & $165.05 \pm 8.46$ & $161.40 \pm 8.13$ \\
\hline $\mathrm{BMI}($ mean $\pm \mathrm{SD})$ & $22.13 \pm 3.04$ & $21.49 \pm 2.77$ \\
\hline \multicolumn{3}{|l|}{ Complications (no.) } \\
\hline Hypertension & 8 & 0 \\
\hline Diabetes mellitus & 2 & 0 \\
\hline \multicolumn{3}{|l|}{ Tumor localization (no.) } \\
\hline Proximal stomach & 15 & 4 \\
\hline Antrum & 14 & 8 \\
\hline Body/fundus & 8 & 8 \\
\hline \multicolumn{3}{|l|}{ Tumor differentiation (no.) } \\
\hline Moderately differentiated & 2 & 5 \\
\hline Moderately-poorly differentiated & 13 & 6 \\
\hline Poorly differentiated & 22 & 7 \\
\hline Unknown & 0 & 2 \\
\hline \multicolumn{3}{|l|}{ Lauren typing (no.) } \\
\hline Intestinal type & 13 & 10 \\
\hline Diffuse type & 11 & 5 \\
\hline Mixed type & 13 & 5 \\
\hline \multicolumn{3}{|l|}{ Tumor stage (no.) } \\
\hline 1 & 4 & 1 \\
\hline II & 12 & 3 \\
\hline III & 21 & 16 \\
\hline
\end{tabular}

Human metabolites are a mixture of products from human genome and bacterial genome, which may be more affected by human genome, but bacterial genome also plays a role in the biosynthesis and degradation of human metabolites. Erawijantari et al. evaluated the influence of gastrectomy as a GC treatment on fecal microbiome and metabolome profiles [16]. Nevertheless, the contributions of microbes to metabolite production and degradation in GC tissues remains unclear.

In this study, we performed 16s rRNA gene sequencing on tumor tissues and matched non-tumor tissues from 37 patients with GC to characterize the gastric microbiota. We also performed untargeted metabolome analysis of the 37 paired GC tissue samples using ultrahigh performance liquid chromatography tandem mass spectrometry (UHPLC-MS/MS) to characterize the gastric metabolome profiles, and combined this analysis with the GC tissue microbiome profiles.

\section{RESULTS}

\section{Altered gastric microbiota in GC tumor tissues compared with matched non-tumor tissues}

As shown in Table S1, the 16s rRNA gene sequencing produced a median of 80,110 clean reads for 37 paired tumor and non-tumor tissues (Cohort 1, Table 1). To measure differences in microbial diversity between the groups, alpha diversity was analyzed. The observed OTUs, which reflects the species richness, was significantly higher in tumor tissues than in non-tumor tissue (464.00 vs. $231.00 ; P<0.001$; Fig. $1 \mathrm{~A}$ ). The Shannon index, which measures species richness and evenness, was also significantly higher in tumor tissues than in non-tumor tissue $(5.20$ vs. 2.98; $P<0.001$; Fig. 1B). To compare the composition of the microbial community between the non-tumor and tumor tissues, we analyzed beta diversity. The weighted UniFrac principal coordinate analysis (PCoA) showed that significant clustering was detected between groups (PERMANOVA, $R^{2}=0.211, P=0.001$, Fig. $1 C$ ). As shown in the Venn diagram, 2222 and 3961 OTUs were detected in the nontumor and tumor tissues, respectively, with 1832 OTUs concurrent in the two groups (Fig. 1D). To identify specific microbial communities associated with GC, we analyzed the composition of the gastric microbiota in non-tumor and tumor tissues using LEfSe analysis. A total of 64 discriminative taxa at all taxonomic levels from phylum to genus were identified (LDA $>3.5, Q<0.05$ ). At the phylum level, the abundance of Proteobacteria was increased in the non-tumor tissues, whereas the abundance of Firmicutes, Bacteroidetes, Actinobacteria, Fusobacteria, and Spirochetes was enriched in the tumor tissues (Fig. 1E). At the genus level, the abundance of Helicobacter was elevated in the nontumor tissues, whereas the abundance of Lactobacillus, Streptococcus, Acinetobacter, Prevotella, Sphingomonas, Bacteroides, Fusobacterium, Comamonas, Empedobacter, and Faecalibacterium was increased in the tumor tissues (Fig. 1E).

\section{Differences in the metabolome profiles between GC tumor and non-tumor tissues}

Since the diversity and composition of the gastric microbiota were different between the non-tumor and tumor tissues from GC patients, we hypothesized that changes in the metabolomic pathways may be partially influenced by gastric microbiota in GC patients. Thus, untargeted metabolome analysis of the tissue samples (37 paired GC tissue samples) was performed using UHPLC-MS/MS, and 1198 metabolites were quantified in the positive and negative modes. The PLS-DA score plot showed that the tumor and non-tumor tissues were separated into two distinct clusters $\left(R^{2} Y=0.89\right.$ and $\left.Q^{2} Y=0.78\right)$ (Fig. 2A). The test for the PLS-DA model showed that the $R^{2}$ value was larger than the $Q^{2}$ value, and that the $Q^{2}$ regression line had a negative intercept $\left(R^{2}=[0.0,0.56], Q^{2}=[0.0,-0.44]\right)$, indicating that the PLS-DA model for this study was valid (Fig. 2B). We observed 150 metabolites with significantly differential relative abundance between the non-tumor and tumor tissues (variable importance in projection (VIP) $>1$ and $Q$ value $<0.05$ and $F C \geq 2$ or $F C \leq 0.5$ ) (Table S2), which included 21 amino acids, 12 carbohydrates and carbohydrate conjugates, 24 fatty acyls, 29 glycerophospholipids, 5 indoles and derivatives, 7 nucleosides, 4 nucleotides, 5 steroids and derivatives, 3 benzenoids, and 2 glycerolipids (Fig. 3). The relative abundance of these metabolites in the classes of amino acids, carbohydrates and carbohydrate conjugates, glycerophospholipids, and nucleosides was higher in the tumor tissues than in the non-tumor tissues (Fig. 3). As for the metabolites of the fatty acyl class, the relative abundance of fatty acid esters of hydroxy fatty acids and prostaglandins was decreased in the tumor tissues compared to the non-tumor tissues. The majority of the remaining metabolites in this class exhibited increased relative abundance in the tumor tissues compared with the nontumor tissues.

\section{Identification of metabolite biomarkers for discriminating tumor from non-tumor tissues}

To identify metabolite biomarkers for discriminating between tumor and non-tumor tissues, we selected the top 15 metabolites according to VIP values (Fig. 4A). Among the 15 metabolites, the relative abundance of 8 metabolites was higher in the tumor tissues than in the non-tumor tissues (Fig. 4B). Next, we preformed the receiver operating curve (ROC) analysis to evaluate the diagnostic accuracy of the 8 metabolites 
(A)

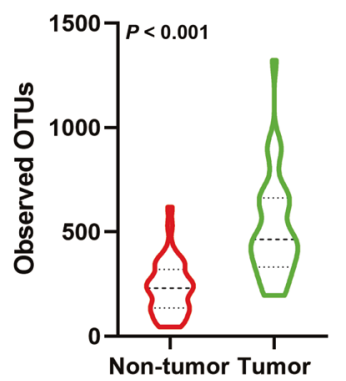

(B)

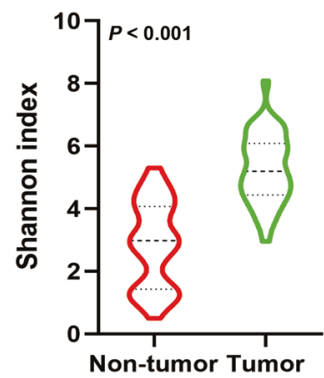

(C)

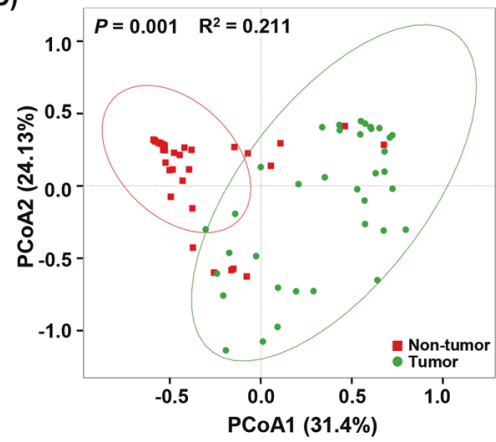

(D)

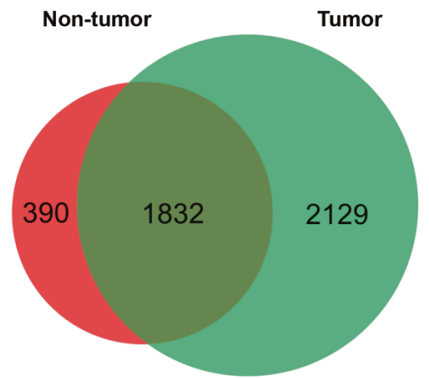

(E)
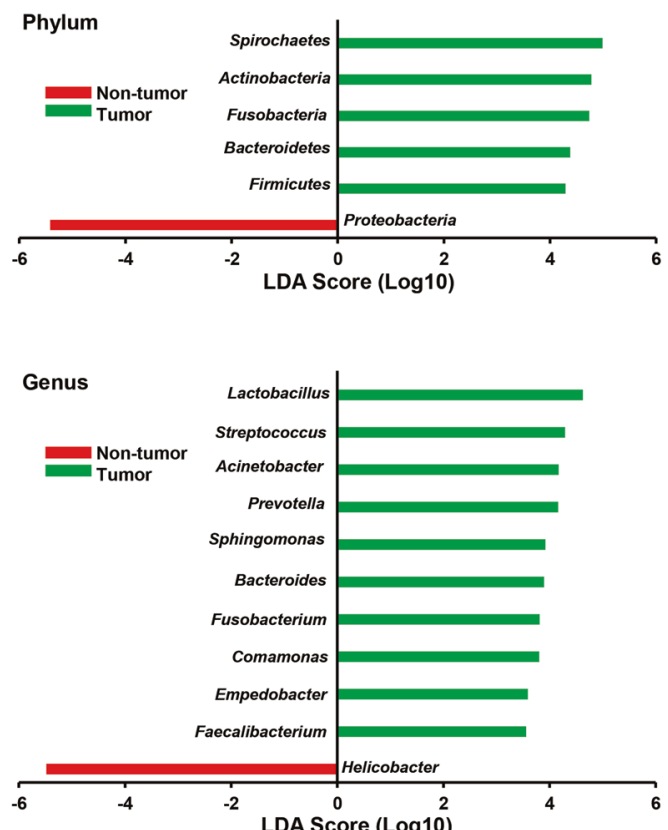

Fig. 1 Altered gastric microbiota in 37 gastric cancer (GC) tissues compared with matched non-tumor tissues. A, B The observed OTUs and Shannon indices were used to evaluate the microbial diversity of the paired tumor and non-tumor tissues. The Wilcoxon matched-pairs signed rank test was performed. C Principal coordinate analysis (PCoA) of the weighted UniFrac distance demonstrated that the non-tumor and tumor tissues showed two distinct clusters. D The Venn diagram illustrates the overlapped OTUs between the paired GC tumor tissues and non-tumor tissues. E Differential taxa at the phylum and genus levels identified by linear discriminant analysis (LDA) effect size (LEfSe) analysis $(\mathrm{LDA}>3.5, Q<0.05)$.

(A)

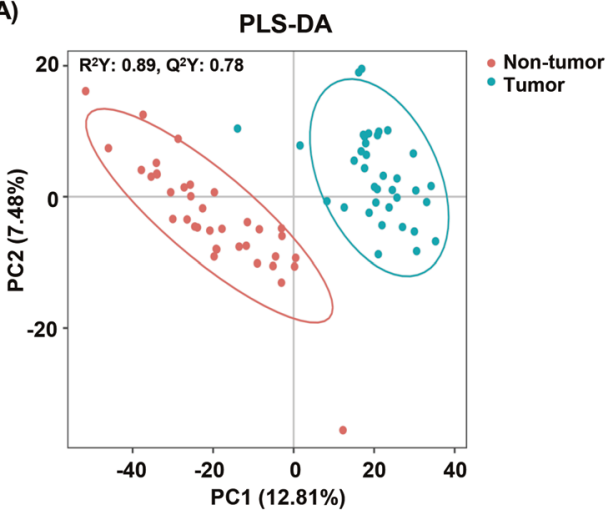

(B)

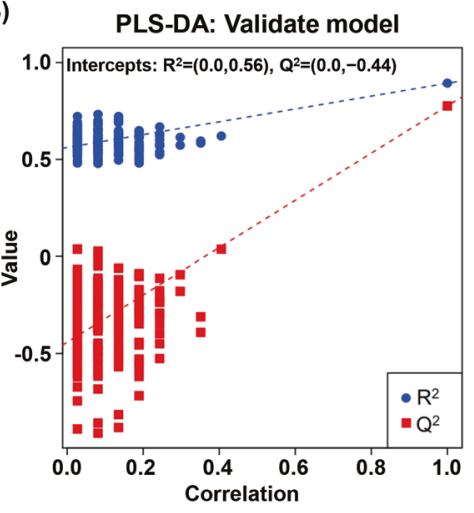

Fig. 2 The metabolome profiles of gastric cancer tissues were different from those of matched non-tumor tissues. A PLS-DA showed that tumor tissues and non-tumor tissues were separated into two distinct clusters. B The test for PLS-DA model showed that the PLS-DA model for this study was valid. PLS-DA, partial least-squares discriminant analysis. QC, quality control. The QC samples were obtained by mixing the equal amounts of metabolites extracted from all samples, which were used for evaluation of the stability of the instrument.

in discriminating between tumor and non-tumor tissues. Metabolites with an area under the curve (AUC) $<0.95$ were eliminated. Finally, we obtained two candidate biomarkers, 1-methylnicotinamide and $\mathrm{N}$-acetyl-D-glucosamine-6-phosphate. Their corresponding AUCs were 0.957 (95\% Cl: 0.917-0.997) and 0.951 (95\% Cl: 0.901-1.000), respectively (Fig. 4C). The AUC for the combination of the two metabolites was $0.976(95 \% \mathrm{Cl}$ : $0.940-1.000$ ) (Fig. 4C). These results suggest that the combination of 1-methylnicotinamide and $\mathrm{N}$-acetyl-D-glucosamine-6phosphate may serve as a potential biomarker for discrimination between GC tumors and non-tumor tissues.
Validation of metabolite biomarkers for discriminating tumor from non-tumor tissues

To validate the combination of 1-methylnicotinamide and $\mathrm{N}$-acetylD-glucosamine-6-phosphate as the biomarkers for discrimination between GC tumors and non-tumor tissues, we recruited 20 additional patients with GC (Cohort 2) for targeted metabolomics (Table 1). As shown in Fig. 5A, B, the concentrations of 1-methylnicotinamide and $N$-acetyl-D-glucosamine-6-phosphate were both significantly higher in GC tumor tissues than in nontumor tissues $(P<0.001)$. The AUCs for 1 -methylnicotinamide and $\mathrm{N}$ acetyl-D-glucosamine-6-phosphate were 0.908 (95\% Cl: 0.794-1.000) 


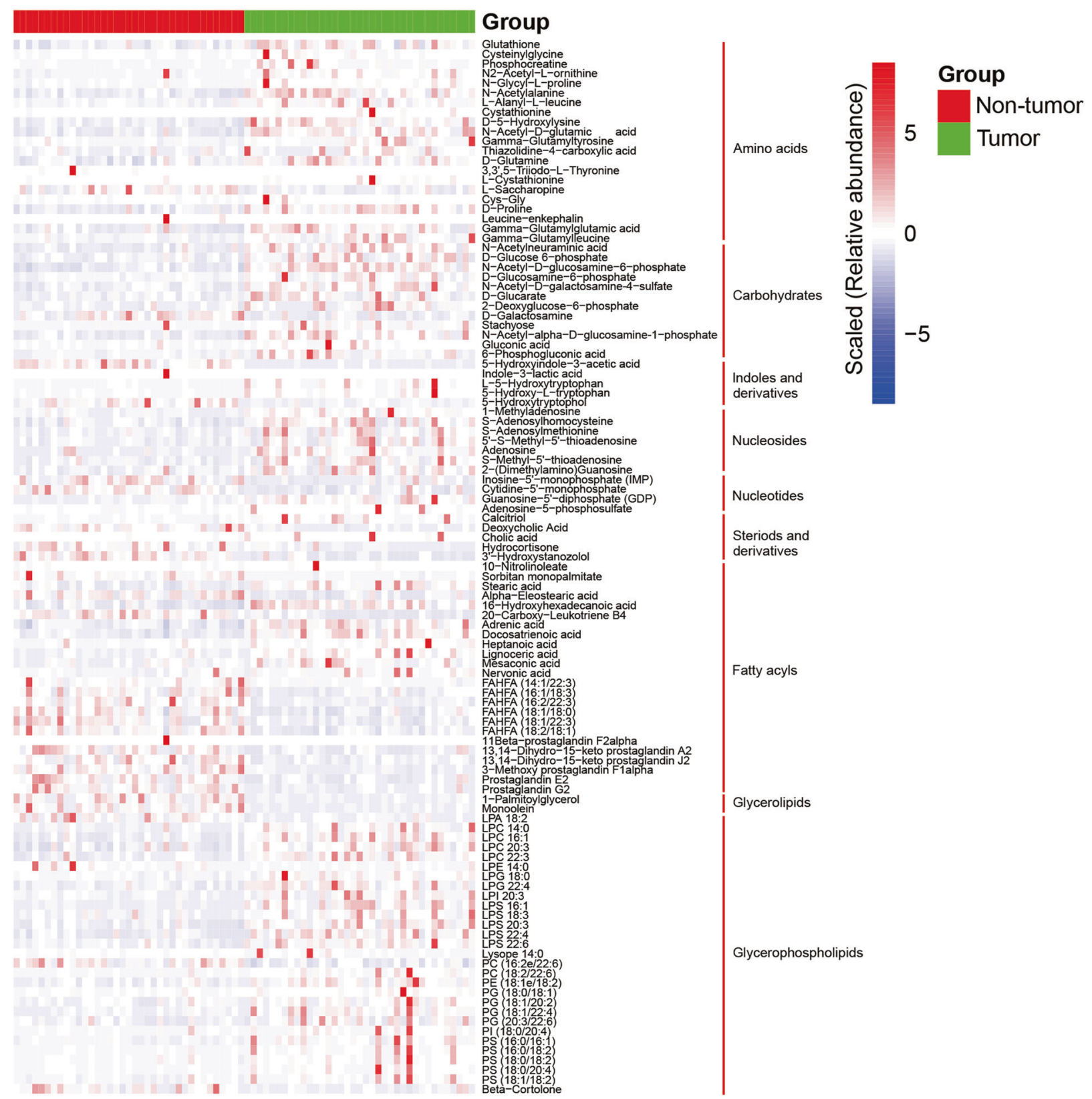

Fig. 3 The heat map shows the differential metabolites between the paired gastric cancer tissues and non-tumor tissues. The heat map shows the scaled relative abundance (Lg) of 109 differential metabolites (VIP $>1$ and $Q$ value $<0.05$ and fold change (FC) $\geq 2$ or FC $\leq 0.5$ ). The discriminative metabolites from top to bottom are amino acids, carbohydrates and carbohydrate conjugates, indoles and derivatives, nucleosides, nucleotides, steroids and derivatives, fatty acyls, glycerolipids, and glycerophospholipids. The differential metabolites were classified using the Human Metabolome Database. $Q$ value, corrected $P$ value.

and 0.835 (95\% Cl: 0.704-0.966), respectively (Fig. 5C, D). The AUC for the combination of the two metabolites was $0.945(95 \% \mathrm{Cl}$ : $0.870-1.000$ ) (Fig. 5E). These results show that the combination of 1-methylnicotinamide and $\mathrm{N}$-acetyl-D-glucosamine-6-phosphate may serve as a robust biomarker for distinction between GC tumors and non-tumor tissues.

\section{KEGG enrichment analysis of differential metabolites}

To determine the main metabolic pathways and signal pathways correlated with the differential metabolites in the non-tumor and tumor tissues, KEGG enrichment analysis was performed. Figure 6A showed 150 discriminative metabolites scattered through multiple pathways, including tryptophan metabolism, amino acid biosynthesis, fatty acid biosynthesis, bile secretion, and galactose metabolism, etc. Furthermore, glutathione, cysteine and methionine metabolism, amino sugar and nucleotide sugar metabolism, and thyroid hormone synthesis pathways were significantly enriched. The pathways of glutathione, cysteine, and methionine metabolism (5 differential metabolites), biosynthesis of amino acids (7 differential metabolites), and bile secretion (6 differential metabolites) contained more differential metabolites than the other pathways.

\section{The association between discriminative genera and metabolites in different pathways}

The Spearman's correlation analysis was used to assess the association between 11 discriminative genera and 25 differential metabolites in the main enriched pathways, which showed that 

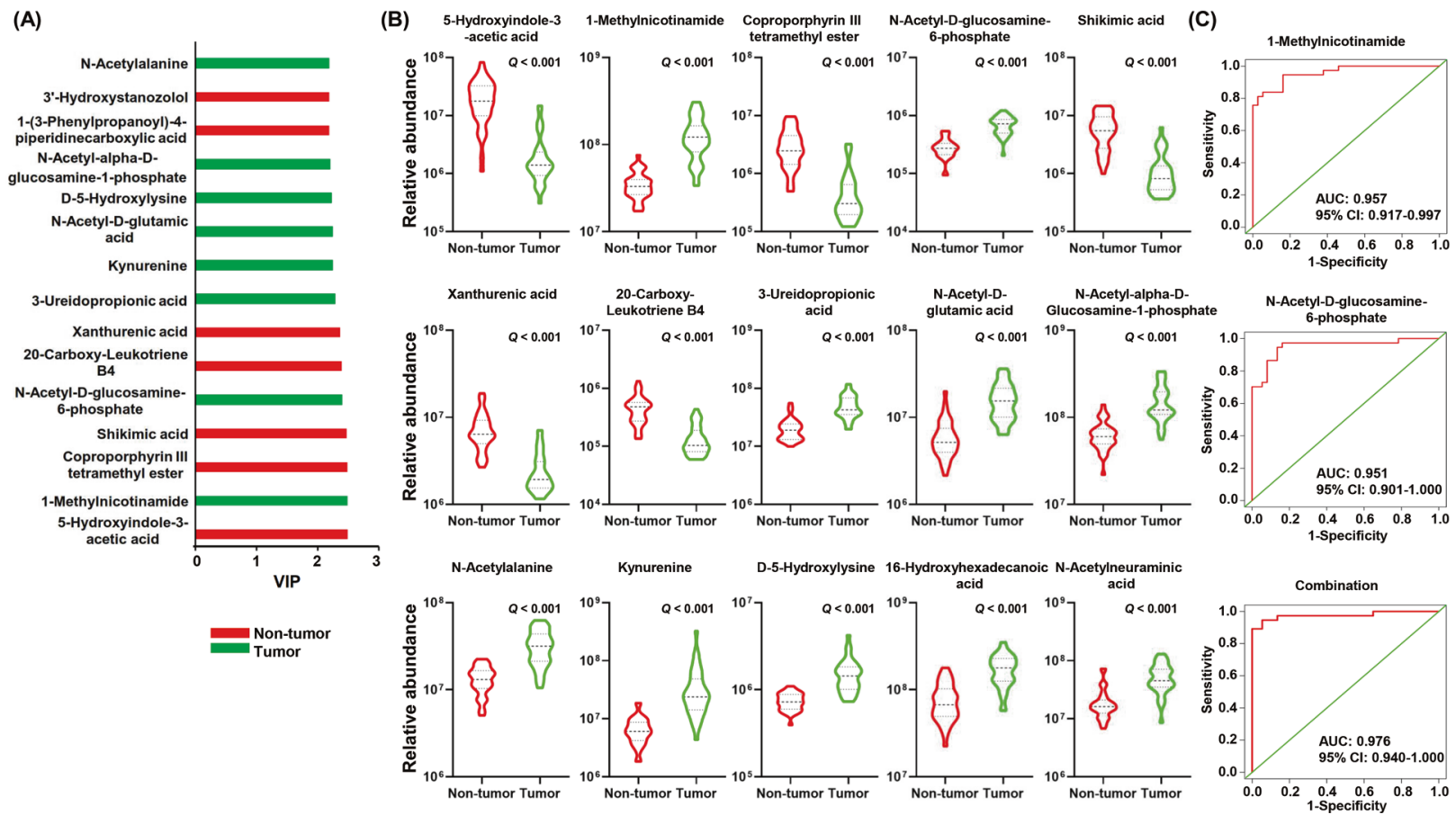

Fig. 4 Identification of metabolite biomarkers for discriminating gastric tumor tissues from non-tumor tissues. A The top 15 metabolites according to VIP values are displayed. VIP, variable importance in projection. B Among the 15 metabolites, the relative abundance of 8 metabolites was higher, but the relative abundance of the rest of the metabolites was lower in the tumor tissues than the non-tumor tissues. $Q$ value, corrected $P$ value. C ROC analysis for 1-methylnicotinamide, $N$-acetyl-D-glucosamine-6-phosphate, and the combination of the two metabolites. ROC, receiver operating curve.

the differential metabolites were not only correlated with the genus Helicobacter but also with other genera (Fig. 6B). Helicobacter was enriched in non-tumor tissues and exhibited a significantly negative correlation with the differential metabolites in pathways of amino sugar and nucleotide sugar metabolism; glutathione, cysteine, and methionine metabolism; and biosynthesis of fatty acids. This indicates that Helicobacter plays a role in the degradation of these metabolites. All differential metabolites in the pathway of amino sugar and nucleotide sugar metabolism were significantly positively associated with Lactobacillus, Streptococcus, Prevotella, Acinetobacter, Comamonas, Empedobacter, and Faecalibacterium. Lactobacillus and Streptococcus both exhibited significantly positive correlation with four out of five differential metabolites in the pathway of glutathione, cysteine, and methionine metabolism. All the differential metabolites in the biosynthesis pathway of fatty acids were significantly positively correlated with Faecalibacterium. Three out of four differential metabolites in this pathway exhibited significantly positive correlation with Lactobacillus and Bacteroides. These results suggested that Lactobacillus, Streptococcus, Prevotella, Acinetobacter, Comamonas, Empedobacter, Faecalibacterium, and Bacteroides contribute greatly to the synthesis of the differential metabolites in the respective pathways. Lactobacillus significantly correlated with all the differential metabolites in the bile secretion pathway, the biosynthesis pathway of amino acids, and the tryptophan metabolism pathway. Helicobacter also showed a significant correlation with several discriminative metabolites in these pathways.

\section{The relationship between discriminative genera and metabolites in different classes}

The analysis of association between differential genera and metabolites in different classes was performed. As shown in Fig. $7 \mathrm{~A}$, Helicobacter significantly correlated with 16 fatty acyls, while
Lactobacillus was significantly associated with 21 fatty acyls, suggesting that the metabolites in this class were strongly influenced by Helicobacter and Lactobacillus. Helicobacter was negatively correlated with the majority of differential metabolites in the classes of amino acids, carbohydrates, nucleosides, nucleotides, and glycerophospholipids; however, Lactobacillus was positively associated with the majority of the differential metabolites in these classes (Fig. 7B-D). These results indicated that Helicobacter and Lactobacillus might contribute to degradation and synthesis of metabolites in these classes, respectively. Bacteroides exhibited significant association with 18 fatty acyls and 19 glycerophospholipids, while Faecalibacterium showed significant correlation with 21 fatty acyls and 22 glycerophospholipids (Fig. 7A, B). These results suggested that Bacteroides and Faecalibacterium might play an important role in synthesis or degradation of fatty acyls and glycerophospholipids. Comamonas showed a significantly positive association with 14 amino acids, indicating a robust contribution of Comamonas to synthesis of amino acids (Fig. 7C). Acinetobacter, Comamonas, Faecalibacterium, Sphingomonas, and Streptococcus were significantly positively associated with 7, 7, 7, 5, and 6 carbohydrates, respectively, which suggested that Acinetobacter, Comamonas, Faecalibacterium, Sphingomonas, and Streptococcus might participate in synthesis of carbohydrates (Fig. 7D). The correlation analysis also showed that Comamonas and Streptococcus might play a role in the synthesis of nucleosides and nucleotides (Fig. 7D).

Microbiota and metabolites associated with clinical features Cohort 1 for microbiome and untargeted metabolome analysis enrolled 16 early-stage (stage I-II) and 21 late-stage (stage III) GC patients (Table 1). The heat map showed that the majority of carbohydrates were gradually increased from non-tumor tissues to early-stage and late-stage tumor tissues (Fig. 8). Particularly, the concentration of $N$-acetyl-D-glucosamine-6-phosphate was 
(A)

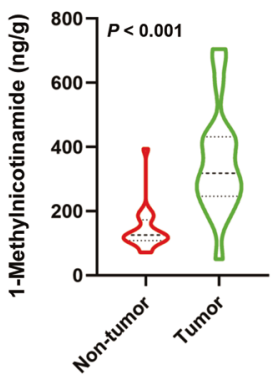

(B)

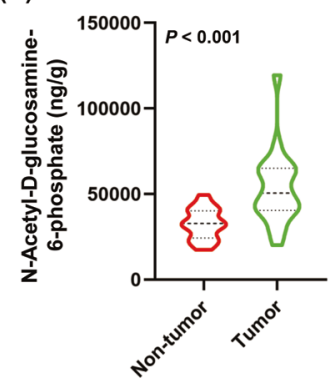

(C)

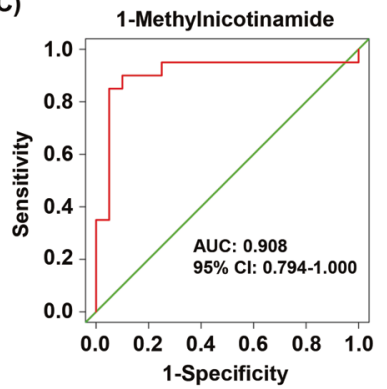

(D)

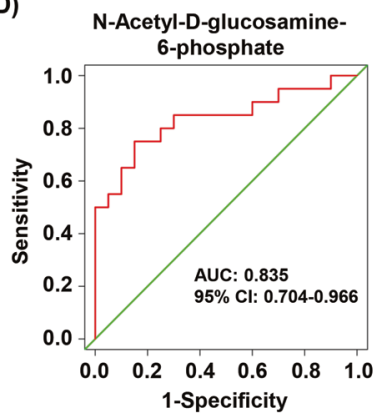

(E)

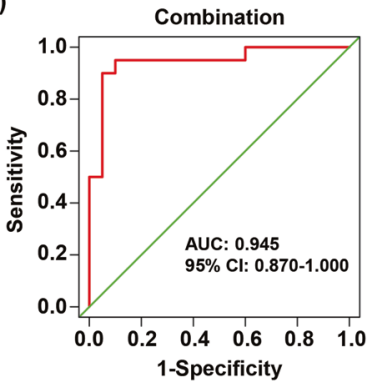

Fig. 5 Validation of metabolite biomarkers for distinguishing between gastric tumor and non-tumor tissues. A The concentrations of 1-methylnicotinamide was compared between tumor tissues and matched non-tumor tissues from 20 patients with gastric cancer (GC). B Comparison of the concentration of $\mathrm{N}$-acetyl-D-glucosamine-6-phosphate between 20 paired GC tumor and non-tumor tissues. C-E ROC analysis for 1-methylnicotinamide (C), N-acetyl-D-glucosamine-6-phosphate (D), and the combination of the two metabolites (E). ROC, receiver operating curve.

gradually elevated from non-tumor tissues to early-stage and latestage tumor tissues with significant difference $(Q<0.05$, Fig. S1). A stepwise increase in the abundance of Acinetobacter, Comamonas, and Sphingomonas from non-tumor tissues to early-stage and latestage tumor tissues was observed (Fig. S2D-F). Nevertheless, the differences were not significant $(Q>0.05)$. However, this trend was not observed for Helicobacter, Lactobacillus, and Streptococcus (Fig. S2A-C). As Figs. S3 and S4 show, no correlation was found between complications and microbiota, and between complications and metabolites.

\section{DISCUSSION}

In this study, the diversity and richness of gastric microbiota was found to be higher in tumor tissues than in non-tumor tissues, which is consistent with the result of a previous study [17]. However, Liu et al. observed decreased diversity and richness in peritumoral and tumoral tissues in comparison to non-tumor tissues from $276 \mathrm{GC}$ patients [10]. There is no consensus on the relationship between microbial diversity and the mucosal tissues of stomach. The relative abundance of Helicobacter was reduced in GC tumor tissues compared to non-tumor tissues, which is consistent with results of two previous studies [10, 17]. The decrease of Helicobacter may be due to the loss of specialized glandular tissues and decreased acid secretion [10].

The abundance of Lactobacillus ranked second in the GC tumor tissues after the abundance of Helicobacter in this study, which differs from previous findings $[10,17]$. However, several studies have found that the proportion of Lactobacillus was higher in GC patients compared to that found in healthy controls [8, 9, 18]. Liu et al. also found that Lactobacillus was higher in GC tumor tissues compared to that in non-tumor tissues [10]. Sonveaux et al. reported that Lactobacillus may produce metabolites that could be used as an energy source for tumor growth and angiogenesis [19]. Previous studies have also shown that the abundance of Streptococcus was increased in GC tumor tissues compared to non-tumor tissues $[10,17]$. The abundance of Streptococcus was found to be elevated in tumor tissues from patients with lung cancer in comparison to non-tumor tissues [20]. Streptococcus displayed a correlation with upregulation of the ERK and PI3K signaling pathways in patients with lung cancer, and in vitro exposure of airway epithelial cells to Streptococcus led to upregulation of these same signaling pathways [21]. We found that Bacteroides exhibited higher abundance in the tumor tissues than in the non-tumor tissues. A restricted gastric microbiota containing only Lactobacillus, Bacteroides, and Clostridium promoted GC development at a similar rate to the complex microbiota in insulin-gastrin transgenic mice [22].

Our microbiome study shares some similarities with the two previous studies, but is also a little different from the two studies $[10,17]$. The study by Shao et al. utilized gastric cardia tissues for microbiome analysis [17], while the study by Liu et al. used proximal stomach, body/fundus, and antrum tissues for research [10]. Our study also used proximal stomach, body/fundus, and antrum tissues. The difference between our study and the study by Liu et al. is that the samples of the study by Liu et al. were mainly from body/fundus, and antrum, while the samples of our study were primarily from proximal stomach, and antrum. Therefore, the differences between the three studies may result from samples with different tumor localization.

Our metabolome analysis of GC tumor tissues and the matched non-tumor tissues revealed 150 differential metabolites, including amino acids, carbohydrates and carbohydrate conjugates, fatty acyls, glycerophospholipids, nucleosides, and nucleotides. Consistent with previous studies $[12,13]$, the majority of the discriminative metabolites in the amino acid class displayed higher relative abundance in the tumor tissues than in the nontumor tissues. Because tumor cells utilize amino acids to generate energy and synthesize proteins and nucleosides, increased concentrations of amino acids are essential for tumor cell proliferation. We also observed enhanced relative abundance of carbohydrates and carbohydrate conjugates in the tumor tissues in comparison to the non-tumor tissues. Elevated glucose uptake is a metabolic feature of tumor cells [23], and carbohydrates and 
(A)

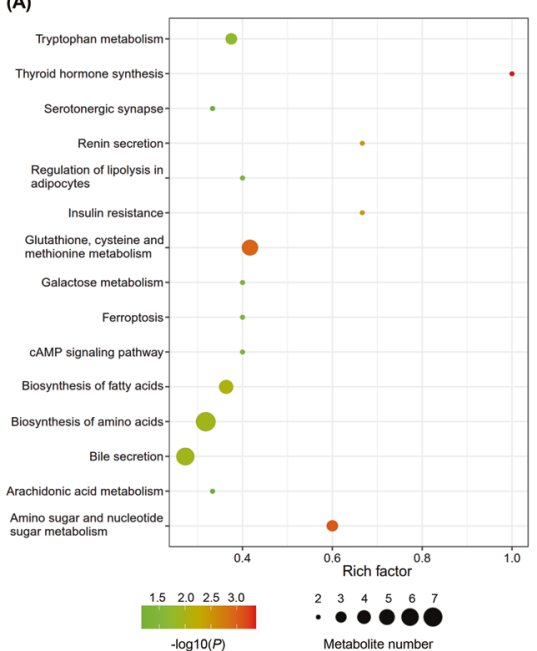

(B)

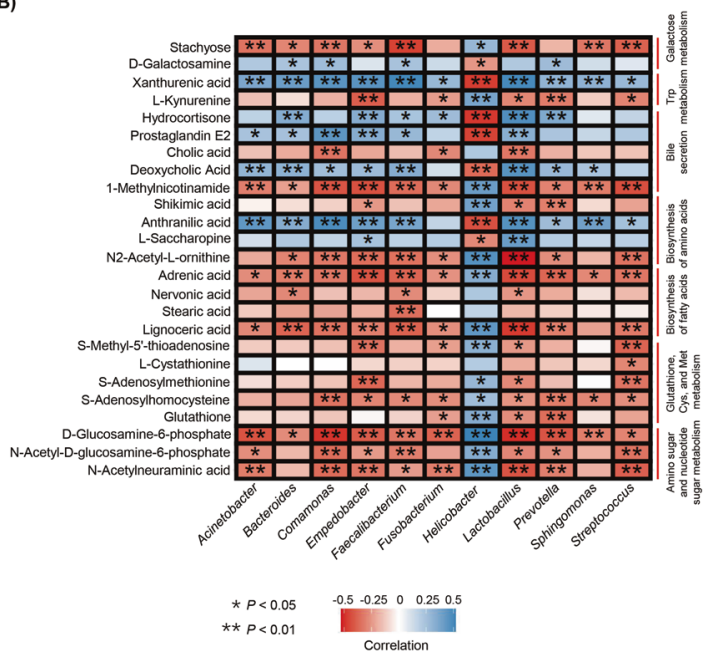

Fig. 6 Pathway analysis for metabolites and integrated analysis of microbiota and metabolites. A The functions of these metabolites and metabolic pathways were studied using the KEGG database, and enriched pathways were displayed by a bubble plot. B The association between 11 discriminative genera and 25 differential metabolites in the main enriched pathways were analyzed using the Spearman's correlation method. Red, positive correlations; blue, negative correlations. ${ }^{*} P$ value $<0.05 ;{ }^{*} P$ value $<0.01$.

carbohydrate conjugates may be used as a source of glucose by tumor cells. Thus, increased carbohydrates and carbohydrate conjugates may be vital in providing enough glucose to satisfy the energy requirements for tumor cell growth. Increased levels of nucleosides were found in the GC tumor tissues in this study, which is consistent with a previous study [12]. Kaji et al. reported that the concentrations of nucleosides were increased in GC patients with peritoneal recurrence compared with those without peritoneal recurrence. It is possible that enhanced levels of nucleosides, especially adenosine, may contribute to a shorter survival in GC patients. Adenosine is a key metabolic and immunecheckpoint regulator that participates in tumor escape from the host immune system [24]. Many therapies targeting adenosine metabolism are in progress. One study found that six glycerophospholipids positively associated with the risk of prostate cancer [25]. Interestingly, we observed 25 glycerophospholipids with increased levels in the GC tumor tissues, which may play important roles in GC development.

KEGG enrichment analysis showed that the pathway of glutathione, cysteine, and methionine metabolism contained 5 metabolites (glutathione, S-adenosylhomocysteine, S-adenosylmethionine, L-cystathionine, and S-methyl-5'-thioadenosine) with significantly increased relative abundance in the GC tumors. Kaji et al. reported that glutathione exhibited a higher level in GC tumor tissues than in the non-tumor tissues [12]. The level of glutathione was also found to be increased in tumor tissues from patients with chromophobe renal cell carcinoma in comparison to non-tumor tissues [26]. Glutathione and cysteine are very important antioxidants, and S-adenosylhomocysteine, S-adenosylmethionine, Lcystathionine, and $S$-methyl-5'-thioadenosine can serve as precursors of glutathione and cysteine. Therefore, the increased levels of metabolites in this pathway could provide GC tumor tissues with robust antioxidation ability. Helicobacter negatively associated with the discriminative metabolites in this pathway, whereas Lactobacillus and Streptococcus displayed a positive correlation with these metabolites, suggesting that Helicobacter, Lactobacillus, and Streptococcus were collectively responsible for the increased relative abundance of the differential metabolites in this pathway. Our data also showed that the levels of the discriminative metabolites in the pathway of fatty acid biosynthesis were upregulated by the collective activity of Helicobacter, Faecalibacterium, Lactobacillus, and Bacteroides. Consistently, we found that Helicobacter,
Lactobacillus, Faecalibacterium, and Bacteroides might be collectively responsible for altered relative abundance of metabolites in the classes of fatty acyls and glycerophospholipids. The elevated relative abundance of the differential metabolites in the pathway of amino sugar and nucleotide sugar metabolism could be due to the collective influence of Helicobacter, Lactobacillus, Streptococcus, Prevotella, Acinetobacter, Comamonas, Empedobacter, and Faecalibacterium, which was in consistence with the observation that Helicobacter, Lactobacillus, Acinetobacter, Comamonas, Faecalibacterium, Sphingomonas, and Streptococcus might be collectively responsible for the synthesis of carbohydrates. Helicobacter and Lactobacillus were negatively and positively associated with the majority of differential metabolites in the classes of amino acids, carbohydrates, nucleosides, nucleotides, and glycerophospholipids, respectively, indicating that Helicobacter and Lactobacillus might contribute to degradation and synthesis of the majority of differential metabolites in these classes, respectively. These results indicated that the metabolome profiles of the GC tumor tissues were strongly influenced by Helicobacter, Lactobacillus, and other microorganisms, which might promote GC development.

Our study had several limitations. First, the sample size is very small, resulting in the lack of significant correlation between clinical features and microbiome, and between clinical features and metabolome. Second, we did not perform longitudinal studies since we could not obtain serial tissue samples from the recruited patients. Third, we utilized PLS-DA, which is highly susceptible to overfitting [27], to characterize the differential metabolites between GC tumor and non-tumor tissues. Thus, the proper model validation was required and we demonstrated that the model was not overfitted. Fourth, the diet could heavily influence both the gastric microbiota and metabolites, but we could not obtain the diet information of patients to analyze the effect of diet on gastric microbiome and metabolome.

In summary, for the first time, we profiled the microbiome and metabolome of tumor tissues and matched non-tumor tissues from GC patients. The diversity and composition of the gastric microbiota were significantly different between the tumor and non-tumor tissues. Helicobacter was enriched in the non-tumor tissues, while Lactobacillus, Streptococcus, Acinetobacter, Prevotella, and six additional genera were enriched in the tumor tissues. The metabolome profiles in the GC tumor tissues were significantly different from those in the matched non-tumor tissues, which may 
(A)

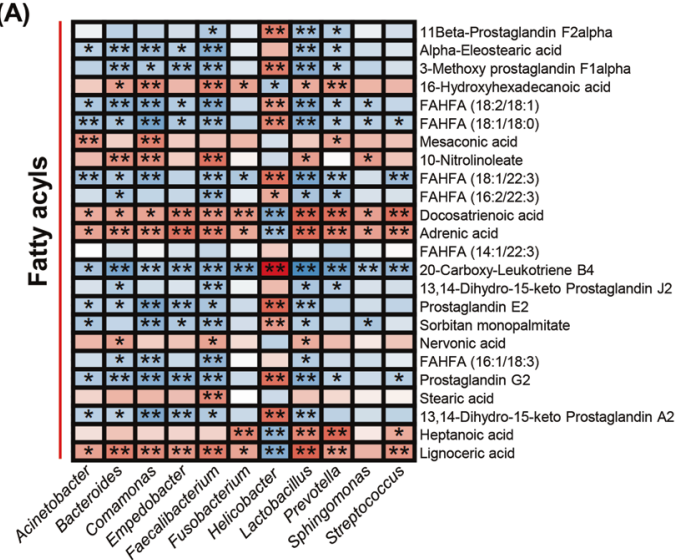

(C)

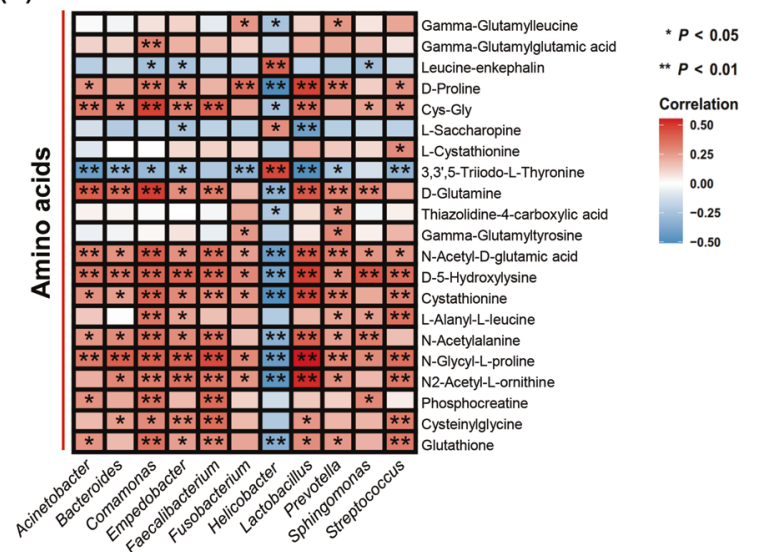

(B)

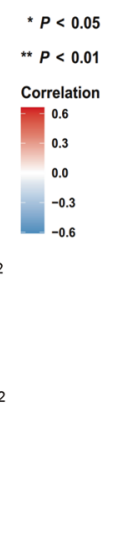

(D)

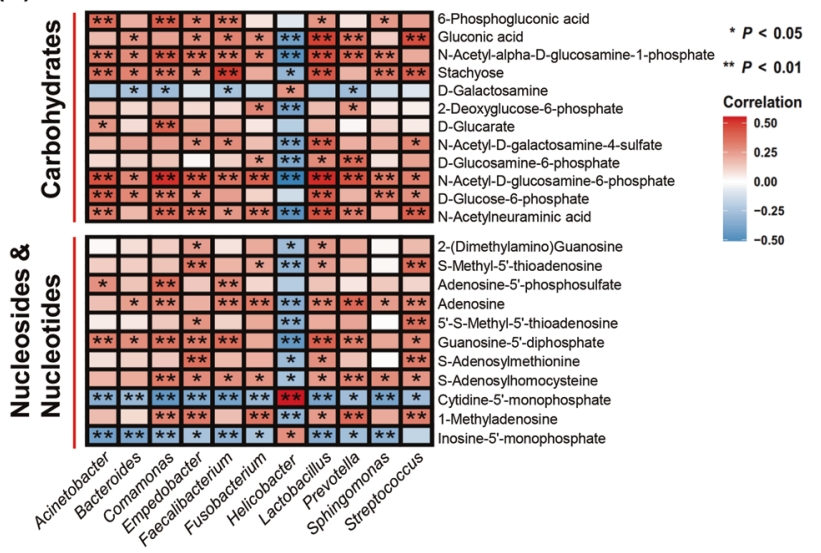

Fig. 7 The analysis of correlation between differential genera and metabolites in different classes. A-D The analysis of correlation between 11 differential genera and discriminative metabolites in the classes of fatty acyls $(n=24)(\mathbf{A})$, differential glycerophospholipids $(n=$ 29) (B), amino acids $(n=21)(\mathbf{C})$, carbohydrates $(n=12)$, nucleosides $(n=7)$, and nucleotides $(n=4)$ (D). Red, positive correlations; blue, negative correlations. ${ }^{*} P$ value $<0.05 ;{ }^{*} P$ value $<0.01$.

be partly due to the collective activities of Helicobacter, Lactobacillus, and other bacteria. The differences in gastric microbiome and metabolome profiles eventually affect GC carcinogenesis and progression. The functions of these microbiota and metabolites are worthy of further research as they may reveal or strengthen a GC therapy.

\section{PATIENTS AND METHODS \\ Patients}

Thirty-seven patients diagnosed with primary GC and undergoing gastrectomy between January 2018 and August 2019, at the First Affiliated Hospital, School of Medicine, Zhejiang University, were enrolled for microbiome and untargeted metabolome analysis. Twenty additional GC patients undergoing gastrectomy between June and August 2021, at The First Affiliated Hospital of Nanchang University, were recruited for targeted metabolome analysis to validate the putative metabolite biomarkers, 1-methylnicotinamide and N-acetyl-D-glucosamine-6-phosphate. All patients received general anesthesia before operation. Most of the patients underwent radical resection of GC, whereas a small number of patients underwent partial gastrectomy. The non-tumor tissue used as the control sample was the gastric mucosal tissue $5 \mathrm{~cm}$ away from the matched tumor tissue. Their general clinical data including age, gender, body mass index (BMI), and histories of hypertension and diabetes were recorded (Table 1). All GC patients were diagnosed by postoperative pathological examinations. The clinical pathological features of GC, such as tumor stage, tumor differentiation and Lauren type of tumor were recorded. The clinical staging was determined according to the 8th edition American Joint Committee on Cancer (AJCC) cancer staging manual of GC TNM Staging. The detailed exclusion criteria were described in supplementary methods and Fig. S5. The study was approved by the Ethics
Committee of the First Affiliated Hospital, School of Medicine, Zhejiang University (2020-IIT-572), and the Medical Research Ethics Committee of the First Affiliated Hospital of Nanchang University (2021-9-001) as per the Declaration of Helsinki. Informed written consent was obtained from all patients before recruitment.

\section{DNA extraction, construction of amplicon library, and sequencing}

The genomic DNA of GC tumor and non-tumor tissues was extracted using cetyltrimethylammonium bromide/sodium dodecyl sulfate method. DNA concentration and purity were obtained by Nanodrop 2000 Spectrophotometer (Thermo Scientific) and $1 \%$ agarose gel electrophoresis. DNA was diluted to $1 \mathrm{ng} / \mu \mathrm{L}$ using sterile water. V3-V4 region of $16 \mathrm{~s}$ rRNA gene was amplified. The primers containing the barcode are $341 \mathrm{~F}$ (CCTAYGGGRBGCASCAG) and 806R (GGACTACNNGGGTATCTAAT). All PCR reactions were performed in $30 \mu \mathrm{L}$ volume, including $15 \mu \mathrm{L}$ of $\mathrm{Q} 5^{\circ}$ HighFidelity 2X Master Mix (New England Biolabs, \# M0492L), $0.2 \mu \mathrm{M}$ of forward and reverse primers, and about $10 \mathrm{ng}$ of template DNA or sterile water (negative control). PCR conditions: initial denaturation at $98^{\circ} \mathrm{C}$ for $1 \mathrm{~min}$, followed by 30 cycles of denaturation at $98^{\circ} \mathrm{C}$ for $10 \mathrm{~s}$, annealing at $50^{\circ} \mathrm{C}$ for $30 \mathrm{~s}$, and extension at $72{ }^{\circ} \mathrm{C}$ for $30 \mathrm{~s}$, final extension at $72^{\circ} \mathrm{C}$ for $5 \mathrm{~min}$. PCR products were analyzed using $2 \%$ agarose gel electrophoresis. According to the manufacturer's recommendation, the Ion Plus Fragment Library Kit 48 rxns (Thermo Scientific, \# 4471252) was used to generate the sequencing library. The library quality was evaluated by the Qubit@ 2.0 Fluorometer (Thermo Scientific). At last, the library was sequenced on the lon $S 5^{\mathrm{TM}}$ XL platform to generate $400-600 \mathrm{bp}$ single-end reads.

\section{Sequencing data analysis}

Filter the raw reads following the Cutadapt quality control process to obtain the high-quality clean reads [28]. Chimera sequences were detected 


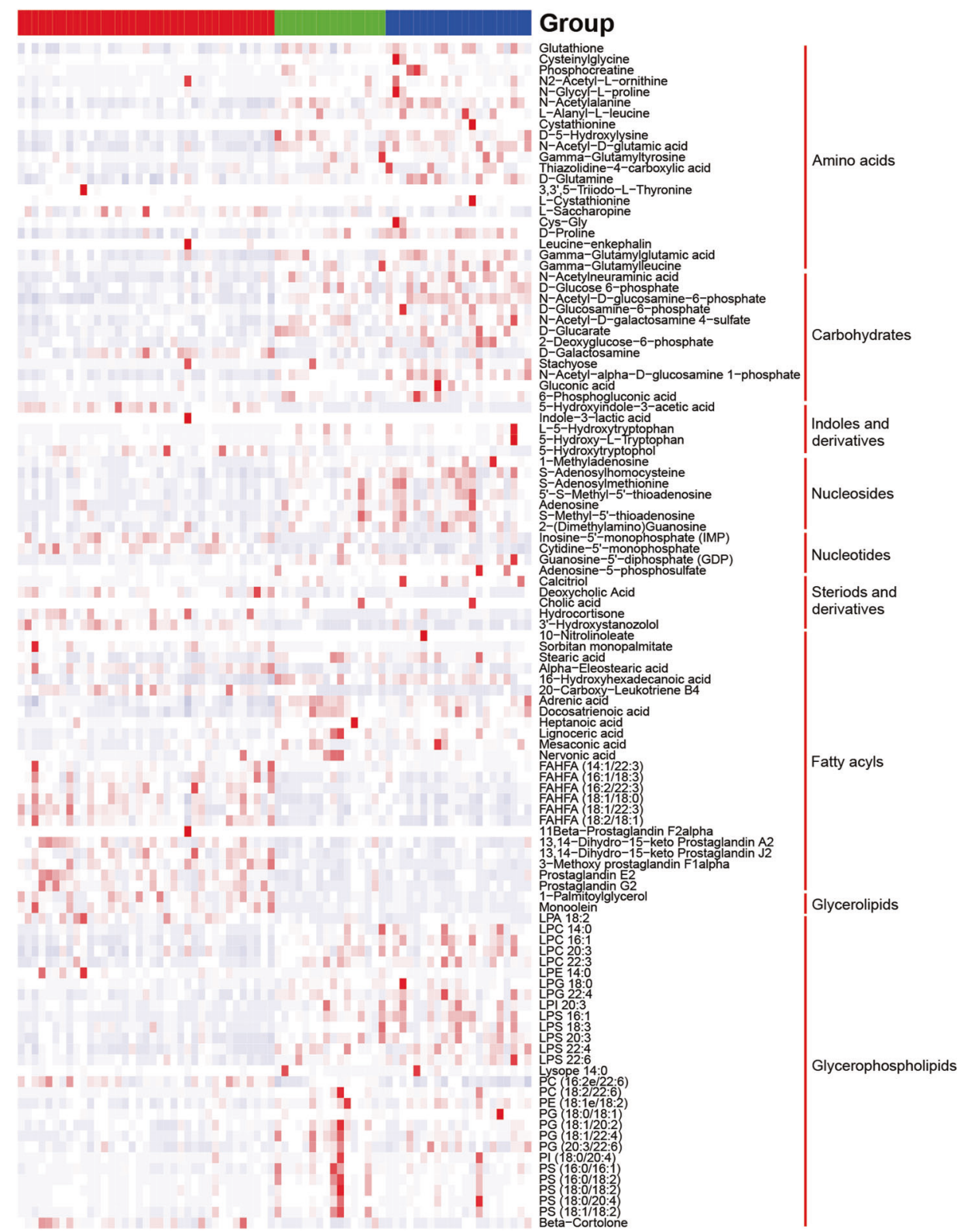

Fig. 8 The heat map shows the association between metabolites and tumor stage. The differences in metabolites among non-tumor tissues $(n=37)$, early-stage (stage I-II, $n=16$ ) and late-stage gastric tumor tissues (stage III, $n=21$ ) were displayed. The heat map shows the scaled relative abundance $(\mathrm{Lg})$ of 109 metabolites.

and removed using the UCHIME algorithm software [29]. Sequences with more than $97 \%$ similarity were allocated to one operational taxonomic unit (OTU) using Uparse software [30]. The taxonomic information was annotated by Silva database based on Mothur algorithm. OTU abundance information was normalized to the sample with the least sequences. Alpha diversity was analyzed using QIIME (Version 1.7.0) to investigate species diversity [31]. QIIME software (Version 1.7.0) was used to calculate the beta diversity based on the weighted Unifrac distance to evaluate differences in microbial community composition. Non-metric multidimensional scaling (NMDS) and principal coordinate analysis (PCoA) were performed. Nonparametric Kruskal-Wallis rank-sum test and the Wilcoxon matchedpairs signed rank test were used to perform linear discriminant analysis (LDA) effect size (LEfSe) analysis to detect discriminative taxa with significant difference between GC tumor and non-tumor tissues.

\section{Tissue sample preparation for untargeted metabolome analysis}

Tissues $(100 \mathrm{mg}$ ) were grounded with liquid nitrogen and the homogenate was resuspended with prechilled $80 \%$ methanol and $0.1 \%$ formic acid (FA) by well vortexing. Then the samples were incubated on ice for $5 \mathrm{~min}$ and were centrifuged at $15,000 \mathrm{rpm}$ for $5 \mathrm{~min}\left(4^{\circ} \mathrm{C}\right)$. The supernatant was diluted to a final concentration containing $53 \%$ methanol by LC-MS grade water. The samples were subsequently transferred to a fresh Eppendorf tube and then centrifuged at $15,000 \mathrm{~g}$ for $10 \mathrm{~min}\left(4^{\circ} \mathrm{C}\right)$. Finally, the supernatant was injected into the UHPLC-MS/MS system.

UHPLC-MS/MS condition for untargeted metabolome analysis UHPLC-MS/MS analysis was performed using a Vanquish UHPLC system coupled with an Orbitrap Q Exactive series mass spectrometer (Thermo Fisher). Samples were injected into a Hyperil Gold column at a flow rate of $0.2 \mathrm{~mL} / \mathrm{min}$. The eluents for the positive polarity mode were eluent $A(0.1 \%$ FA in water) and eluent $B$ (methanol).The eluents for the negative polarity mode were eluent $A(5 \mathrm{mM}$ ammonium acetate, $\mathrm{pH} 9.0)$ and eluent $B$ (methanol).The solvent gradient was set as follows: $2 \% \mathrm{~B}, 1.5 \mathrm{~min} ; 2-100 \%$ B, $12.0 \mathrm{~min} ; 100 \% \mathrm{~B}, 14.0 \mathrm{~min} ; 100-2 \% \mathrm{~B}, 14.1 \mathrm{~min} ; 2 \% \mathrm{~B}, 17 \mathrm{~min}$. The $\mathrm{Q}$ Exactive mass spectrometer worked under positive and negative polarity mode, the spray voltage was $3.2 \mathrm{kV}$, and the capillary temperature was $320^{\circ} \mathrm{C}$. 


\section{The conditions for targeted metabolome}

The mixed standard solution of $\mathrm{N}$-acetyl-D-glucosamine-6-phosphate disodium salt (J\&K Scientific) with a concentration of $5 \mathrm{mg} / \mathrm{mL}$ and 1-methylnicotinamide chloride (Sigma Aldrich) with a concentration of $2 \mathrm{mg} / \mathrm{mL}$ was prepared. The creatinine-d3 (Shanghai ZZBio) was used as the internal standard.

Tissues $(50 \mathrm{mg})$ were grounded with liquid nitrogen and the homogenate was resuspended with $150 \mu \mathrm{L}$ of prechilled $80 \%$ methanol including creatinine- $\mathrm{d} 3(200 \mathrm{ng} / \mathrm{mL})$ by well vortexing. Then the samples were incubated on ice for $5 \mathrm{~min}$ and were centrifuged at 12,000 rpm for 10 min $\left(4^{\circ} \mathrm{C}\right)$. The supernatant was injected into the HPLC-MS/MS system. HPLC-MS/MS analysis was performed using an ExionLC ${ }^{T M}$ AD HPLC system coupled with a QTRAP 6500 plus mass spectrometer (AB Sciex). Samples were injected into an ACQUITY UPLC HSS T3 column at a flow rate of $0.3 \mathrm{~mL} / \mathrm{min}$. The eluents for HPLC were eluent $\mathrm{A}(0.1 \% \mathrm{FA}$ in water, $10 \mathrm{mM}$ ammonium acetate) and eluent $B$ (methanol). The solvent gradient was set as follows: $2 \%$ B, 1.0 min; 2-100\% B, 1.5 min; $100 \%$ B, 2.0 min; 100$2 \%$ B, $2.1 \mathrm{~min} ; 2 \%$ B, $3.0 \mathrm{~min}$. A QTRAP 6500 plus mass spectrometer equipped with electrospray ionization source was operated in positive/ negative ion mode with a spray voltage of $4500 \mathrm{~V}$, and ion source temperature of $550^{\circ} \mathrm{C}$. The pressure of curtain gas, ion source gas 1 and 2 were 35,60 , and $60 \mathrm{psi}$, respectively.

The standard solution was diluted in gradient, and the standard solutions with different concentrations were detected by HPLC-MS. The standard curve was plotted with the concentration of the standard as the abscissa and the ratio of the peak area of the standard to that of the internal standard as the ordinate.

\section{Statistical analysis}

The statistical analyses were performed using GraphPad Prism (Version 8.0; GraphPad Software) software. Statistical significance was defined as a twosided $P$ value of $<0.05$. The Wilcoxon matched-pairs signed rank test was used to calculate the difference in observed species, Shannon index, the abundance of taxa, and the concentration of metabolites between groups. $P$ values were corrected using Benjamini-Hochberg method, and the corrected $P$ values were denoted as $Q$ values. Microbiome-metabolome correlation analysis was performed using Spearman's correlation method and displayed using R software (Version 3.6.1).

\section{DATA AVAILABILITY}

Raw sequence data of $16 \mathrm{~s}$ rRNA microbiome have been deposited in the China National Microbiological Data Center (Project accession number NMDC10017675 and microbiome accession numbers NMDC40001044 to NMDC40001117). The corresponding author has access to all data in the study.

\section{REFERENCES}

1. Bray F, Ferlay J, Soerjomataram I, Siegel RL, Torre LA, Jemal A. Global cancer statistics 2018: GLOBOCAN estimates of incidence and mortality worldwide for 36 cancers in 185 countries. CA Cancer J Clin. 2018;68:394-424.

2. Chen W, Zheng R, Baade PD, Zhang S, Zeng H, Bray F, et al. Cancer statistics in China, 2015. CA Cancer J Clin. 2016;66:115-32.

3. Chen Y, Segers S, Blaser MJ. Association between Helicobacter pylori and mortality in the NHANES III study. Gut. 2013;62:1262-69.

4. Naylor G, Axon A. Role of bacterial overgrowth in the stomach as an additional risk factor for gastritis. Can J Gastroenterol. 2003;17:13b-7b. Suppl B.

5. Qin J, Li R, Raes J, Arumugam M, Burgdorf KS, Manichanh C, et al. A human gut microbial gene catalogue established by metagenomic sequencing. Nature. 2010;464:59-65.

6. Coker OO, Dai Z, Nie Y, Zhao G, Cao L, Nakatsu G, et al. Mucosal microbiome dysbiosis in gastric carcinogenesis. Gut. 2018;67:1024-32.

7. Gantuya B, El Serag HB, Matsumoto T, Ajami NJ, Uchida T, Oyuntsetseg K, et al. Gastric mucosal microbiota in a Mongolian population with gastric cancer and precursor conditions. Aliment Pharm Ther. 2020;51:770-80.

8. Castaño-Rodríguez N, Goh KL, Fock KM, Mitchell HM, Kaakoush NO. Dysbiosis of the microbiome in gastric carcinogenesis. Sci Rep. 2017;7:15957.

9. Eun CS, Kim BK, Han DS, Kim SY, Kim KM, Choi BY, et al. Differences in gastric mucosal microbiota profiling in patients with chronic gastritis, intestinal metaplasia, and gastric cancer using pyrosequencing methods. Helicobacter. 2014;19:407-16.

10. Liu X, Shao L, Liu X, Ji F, Mei Y, Cheng Y, et al. Alterations of gastric mucosal microbiota across different stomach microhabitats in a cohort of 276 patients with gastric cancer. EBioMedicine. 2019;40:336-48.
11. Louis $\mathrm{P}$, Hold GL, Flint HJ. The gut microbiota, bacterial metabolites and colorectal cancer. Nat Rev Microbiol. 2014;12:661-72.

12. Kaji $S$, Irino $T$, Kusuhara $M$, Makuuchi R, Yamakawa $Y$, Tokunaga $M$, et al. Metabolomic profiling of gastric cancer tissues identified potential biomarkers for predicting peritoneal recurrence. Gastric Cancer. 2020;23:874-83.

13. Hirayama A, Kami K, Sugimoto $M$, Sugawara $M$, Toki N, Onozuka H, et al. Quantitative metabolome profiling of colon and stomach cancer microenvironment by capillary electrophoresis time-of-flight mass spectrometry. Cancer Res. 2009;69:4918-25.

14. Song $H$, Wang L, Liu HL, Wu XB, Wang HS, Liu ZH, et al. Tissue metabolomic fingerprinting reveals metabolic disorders associated with human gastric cancer morbidity. Oncol Rep. 2011;26:431-8.

15. Wu H, Xue R, Tang Z, Deng C, Liu T, Zeng H, et al. Metabolomic investigation of gastric cancer tissue using gas chromatography/mass spectrometry. Anal Bioanal Chem. 2010;396:1385-95.

16. Erawijantari PP, Mizutani S, Shiroma H, Shiba S, Nakajima T, Sakamoto T, et al. Influence of gastrectomy for gastric cancer treatment on faecal microbiome and metabolome profiles. Gut. 2020;69:1404-15.

17. Shao D, Vogtmann E, Liu A, Qin J, Chen W, Abnet CC, et al. Microbial characterization of esophageal squamous cell carcinoma and gastric cardia adenocarcinoma from a high-risk region of China. Cancer. 2019;125:3993-4002.

18. Aviles-Jimenez F, Vazquez-Jimenez F, Medrano-Guzman R, Mantilla A, Torres J. Stomach microbiota composition varies between patients with non-atrophic gastritis and patients with intestinal type of gastric cancer. Sci Rep. 2014;4:4202.

19. Sonveaux P, Copetti T, De Saedeleer CJ, Végran F, Verrax J, Kennedy KM, et al. Targeting the lactate transporter MCT1 in endothelial cells inhibits lactateinduced HIF-1 activation and tumor angiogenesis. PLoS ONE. 2012;7:e33418.

20. Liu HX, Tao LL, Zhang J, Zhu YG, Zheng Y, Liu D, et al. Difference of lower airway microbiome in bilateral protected specimen brush between lung cancer patients with unilateral lobar masses and control subjects. Int J Cancer. 2018;142:769-78.

21. Tsay JJ, Wu BG, Badri MH, Clemente JC, Shen N, Meyn P, et al. Airway microbiota is associated with upregulation of the PI3K pathway in lung cancer. Am J Respir Crit Care Med. 2018;198:1188-98.

22. Lertpiriyapong K, Whary MT, Muthupalani S, Lofgren JL, Gamazon ER, Feng Y, et al. Gastric colonisation with a restricted commensal microbiota replicates the promotion of neoplastic lesions by diverse intestinal microbiota in the Helicobacter pylori INS-GAS mouse model of gastric carcinogenesis. Gut. 2014;63:54-63.

23. Zambell KL, Fitch MD, Fleming SE. Acetate and butyrate are the major substrates for de novo lipogenesis in rat colonic epithelial cells. J Nutr. 2003;133:3509-15.

24. Boison D, Yegutkin GG. Adenosine metabolism: emerging concepts for cancer therapy. Cancer Cell. 2019;36:582-96.

25. Röhnisch HE, Kyrø C, Olsen A, Thysell E, Hallmans G, Moazzami AA. Identification of metabolites associated with prostate cancer risk: a nested case-control study with long follow-up in the Northern Sweden Health and Disease Study. BMC Med. 2020;18:187.

26. Xiao Y, Clima R, Busch J, Rabien A, Kilic E, Villegas SL, et al. Decreased mitochondrial DNA content drives OXPHOS dysregulation in chromophobe renal cell carcinoma. Cancer Res. 2020;80:3830-40.

27. Szymańska E, Saccenti E, Smilde AK, Westerhuis JA. Double-check: validation of diagnostic statistics for PLS-DA models in metabolomics studies. Metabolomics. 2012;8:3-16. Suppl 1.

28. Martin M. Cutadapt removes adapter sequences from high-throughput sequencing reads. EMBnet J. 2011;17:10.

29. Edgar RC, Haas BJ, Clemente JC, Quince C, Knight R. UCHIME improves sensitivity and speed of chimera detection. Bioinformatics. 2011;27:2194-200.

30. Edgar RC. UPARSE: highly accurate OTU sequences from microbial amplicon reads. Nat Methods. 2013;10:996-8.

31. Caporaso JG, Kuczynski J, Stombaugh J, Bittinger K, Bushman FD, Costello EK, et al. QIIME allows analysis of high-throughput community sequencing data. Nat Methods. 2010;7:335-6.

\section{ACKNOWLEDGEMENTS}

The authors thank the study participants and the clinical staff who supported this study. The authors would like to thank Editage for English language editing.

\section{AUTHOR CONTRIBUTIONS}

D.D., Y.Y., L.T., J.Y. (Jing Ye), and H.J. designed the study; Y.Y., W.Q., L.T., and J.Y. (Jieqing Yu) collected samples; D.D., Y.Y., L.T., and T.D. performed the experiments; D.D. performed analysis and interpretation, and drafted the manuscript; H.J., J.Y., and L.T. supervised the study. All authors contributed to manuscript revision, and read and approved the submitted version. 


\section{FUNDING}

This study was supported by the National Natural Science Foundation of China (No. 81860182 and No. 82160527), the Natural Science Foundation of Jiangxi Province (No. 20181BAB205036), Project of the Regional Diagnosis and Treatment Center of the Health Planning Committee (No. JBZX-201903), China Postdoctoral Science Foundation (2018M633215), Science and Technology Department of Jiangxi Province (No. 20203BBGL73201), Health Commission of Jiangxi Province (No. 20203229), and The First Affiliated Hospital of Nanchang University (No. YFYPY202002).

\section{COMPETING INTERESTS}

The authors declare no competing interests.

\section{ETHICS APPROVAL AND CONSENT TO PARTICIPATE}

The study was approved by the Ethics Committee of the First Affiliated Hospital, School of Medicine, Zhejiang University (2020-IIT-572), and the Medical Research Ethics Committee of the First Affiliated Hospital of Nanchang University (2021-9-001) as per the Declaration of Helsinki. Informed written consent was obtained from all patients before recruitment.

\section{ADDITIONAL INFORMATION}

Supplementary information The online version contains supplementary material available at https://doi.org/10.1038/s41419-021-04396-y.
Correspondence and requests for materials should be addressed to Daofeng Dai, Lisong Teng, Jing Ye or Hongqun Jiang.

Reprints and permission information is available at http://www.nature.com/ reprints

Publisher's note Springer Nature remains neutral with regard to jurisdictional claims in published maps and institutional affiliations.

Open Access This article is licensed under a Creative Common adaptation, distribution and reproduction in any medium or format, as long as you give appropriate credit to the original author(s) and the source, provide a link to the Creative Commons license, and indicate if changes were made. The images or other third party material in this article are included in the article's Creative Commons license, unless indicated otherwise in a credit line to the material. If material is not included in the article's Creative Commons license and your intended use is not permitted by statutory regulation or exceeds the permitted use, you will need to obtain permission directly from the copyright holder. To view a copy of this license, visit http://creativecommons. org/licenses/by/4.0/.

(C) The Author(s) 2021 